\title{
In Vitro Evaluation of Cell-Seeded Chitosan Films for Peripheral Nerve Tissue Engineering
}

\author{
Sandra Wrobel, MSc, ${ }^{1,2}$ Sofia Cristina Serra, BSc, ${ }^{3,4}$ Silvina Ribeiro-Samy, BSc, ${ }^{3,4}$ Nuno Sousa, PhD, 3,4 \\ Claudia Heimann, Dr rer nat, ${ }^{5}$ Christina Barwig, Dr rer nat, ${ }^{5}$ Claudia Grothe, Dr rer nat, PhD, ${ }^{1,2}$ \\ Antonio Jose Salgado, $\mathrm{PhD}{ }^{3,4, \star}$ and Kirsten Haastert-Talini, DVM, PhD ${ }^{1,2, *}$
}

Natural biomaterials have attracted an increasing interest in the field of tissue-engineered nerve grafts, representing a possible alternative to autologous nerve transplantation. With the prospect of developing a novel entubulation strategy for transected nerves with cell-seeded chitosan films, we examined the biocompatibility of such films in vitro. Different types of rat Schwann cells (SCs) - immortalized, neonatal, and adult—as well as rat bone-marrow-derived mesenchymal stromal cells (BMSCs) were analyzed with regard to their cell metabolic activity, proliferation profiles, and cell morphology after different time points of mono- and cocultures on the chitosan films. Overall the results demonstrate a good cytocompatibility of the chitosan substrate. Both cell types were viable on the biomaterial and showed different metabolic activities and proliferation behavior, indicating cell-type-specific cell-biomaterial interaction. Moreover, the cell types also displayed their typical morphology. In cocultures adult SCs used the BMSCs as a feeder layer and no negative interactions between both cell types were detected. Further, the chitosan films allow neurite outgrowth from dissociated sensory neurons, which is additionally supported on film preseeded with SC-BMSC cocultures. The presented chitosan films therefore demonstrate high potential for their use in tissue-engineered nerve grafts.

\section{Introduction}

C OMPLETE TRANSECTION INJURY of a peripheral nerve results in denervation of target tissue with loss of sensation and motor control. A yearly occurrence of peripheral nerve injuries of 300,000 cases has been estimated for Europe. ${ }^{1}$ Complete nerve transection injuries need surgical intervention, a treatment that is reported for more than 50,000 cases per year in the United States while the estimated number of unreported cases is much higher. ${ }^{2}$ The surgical reconstruction of peripheral nerve continuity has to be performed in a tension-free coaptation, which is only possible for small nerve gaps. When extended nerve gaps are reconstructed by end-to-end suture, tension and drag force will create reactive fibrosis and impair regrowth of nerve fibers. ${ }^{3}$ The clinical gold standard for bridging peripheral nerve gaps of critical length is the transplantation of autologous nerve tissue, which is harvested from sensory nerves (e.g., sural nerve) of the same patient. ${ }^{4}$ In general, autologous nerve grafts provide optimal guidance cues for regenerating nerve fibers, such as peripheral glia cells and
Schwann cells (SCs), which do not only produce regeneration-promoting factors but proliferate and also line their basal laminae up to the so-called bands of Büngner, the guiding tubes within the distal nerve segments. ${ }^{5}$

Admittedly the use of autologous nerve tissue leads to loss of sensation at the site of harvest and the functional outcome after its utilization is described to range from extremely poor ${ }^{6}$ to highly satisfying. ${ }^{2}$ As an alternative to this, entubulation strategies, using biomaterials, have been performed. However, so far the results have been quite disappointing.

To tackle this, new biomaterials have been developed that can easily be modified to provide an optimized environment for peripheral nerve regeneration and qualify as substitutes for autologous nerve grafts. Chitosan is among the biodegradable polymers that demonstrate good properties for neural tissue engineering. ${ }^{7}$ It is an attractive material to produce the outer shell of new nerve grafts because it can easily be blended with other materials or modified on its surface to provide guiding and structural cues supporting axonal regrowth. ${ }^{8}$

\footnotetext{
${ }^{1}$ Hannover Medical School, Institute of Neuroanatomy, Hannover, Germany.

${ }^{2}$ Center for Systems Neuroscience (ZSN), Hannover, Germany.

${ }^{3}$ Life and Health Sciences Research Institute (ICVS), School of Health Sciences, University of Minho, Braga, Portugal.

${ }^{4}$ ICVS/3B's, PT Government Associate Laboratory, Braga/Guimarães, Portugal.

${ }^{5}$ Medovent GmbH, Mainz, Germany.

*These authors contributed equally to this work and share senior authorship.
} 
In this study we investigated the properties of films made of chitosan in order to produce the outer scaffold of nerve grafts. Chitosan films can be rolled to form hollow tubes and the respective surface modifications will allow the fabrication of tailor-made peripheral nerve grafts. Figure 1 summarizes these future perspectives for the use of chitosan films.

To tailor the biodegradability of chitosan materials, the degree of acetylation (DA) can be modified (Fig. 1A, chitosan molecule with one acetylation group) by modifying the number of acetylation groups conjugated to the chitosan. Further, the films can be seeded with regenerationsupporting cell types, such as cocultures of SCs and bonemarrow-derived mesenchymal stromal cells (BMSCs) (Fig. 1B). Both cell types qualify as cellular substitute to tissueengineered nerve grafts for different reasons. SCs are crucially involved into the set-up of the pro-regeneration milieu within the distal nerve segments and the potential of nerve autotransplantation is mainly attributed to the presence of this cell type within the grafts. ${ }^{5,9}$ BMSCs have been described as attributable to in vitro transdifferentiation into SC-like cells, ${ }^{10,11}$ a feature that would allow to include SC properties with the benefit of avoiding autologous SC harvest. Additionally, despite their putative potential for transdifferentiation, mesenchymal stromal cells could serve as living mini-pumps for regeneration-promoting factors. Indeed the secretome of these cells possesses several growth factors with strong implications for nervous tissue repair, as reviewed by Teixeira et al. ${ }^{12}$ Because both SCs and BMSCs are potential candidates for peripheral nerve tissue engineering, we tested in this study the cell-biomaterial interactions of both cell types separately as well as in cocultures.

\section{Materials and Methods}

\section{Manufacturing of chitosan films}

Chitosan films were produced at Medovent $\mathrm{GmbH}$. Briefly, highly purified chitosan with a DA of 5\% (Altakitin S.A.) was dissolved in $0.75 \%$ acetic acid to obtain a $1.5 \%$ solution, filtered, and poured into Petri dishes, followed by drying at room temperature (RT). The resulting films were treated with a solution of ammonia in methanol/water, ${ }^{13}$ followed by intense washing with distilled water, and drying. Finally, the films were cut into the required size and sterilized by electron beam. The 5\% DA was chosen because in another study, focusing on in vivo studies of hollow nonfunctionalized chitosan tubes, this DA demonstrated the best support for the regenerating peripheral nerve tissue. ${ }^{7}$

\section{Characterization of chitosan films}

Mechanical properties and dimensions. Films were cut in stripes of $\sim 5-\mathrm{mm}$ width. Tensile strength and Young's modulus were determined using a mechanical tester (model Z3; Thümler $\mathrm{GmbH}$ ) and the software Zpm_v4-5. Film thickness was determined using a caliper (DURATOOL).

Scanning electron microscopy. To characterize their surface, chitosan films were observed by scanning electron microscopy, with a Leica Cambridge S360 (Leica Cambridge). All the samples were previously gold-coated in a Sputter Jeol JFC 1100 equipment.

Fourier transform infrared spectroscopy. Fourier transform infrared (FTIR) spectrum was collected using Shimadzu IRPrestige 21 and samples were prepared as potassium bromide pellets (KBr; Pike Technologies) at ambient temperature $\left(25^{\circ} \mathrm{C}\right)$. The spectrum was collected using 32 scans with a resolution of $4 \mathrm{~cm}^{-1}$.

\section{Cell culture}

Rat neonatal SCs. Neonatal SCs (neoSCs) were harvested from Wistar rat pups (P1-P3). Enzymatic digestion of isolated sciatic nerves according to Haastert et al. ${ }^{14}$ was stopped after $50 \mathrm{~min}$ with cell culture medium (DMEM, $0.1 \mathrm{mM}$ Forskolin, $1 \%$ Pen/strep, $2 \mathrm{mM}$ L-glutamine, $1 \mathrm{mM}$ sodium pyruvate, and $10 \%$ fetal calf serum [FCS]; all from
A
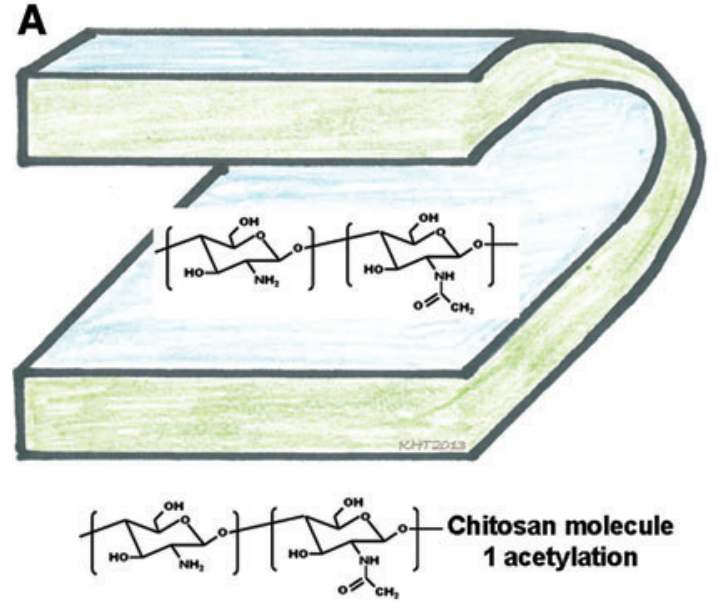

B

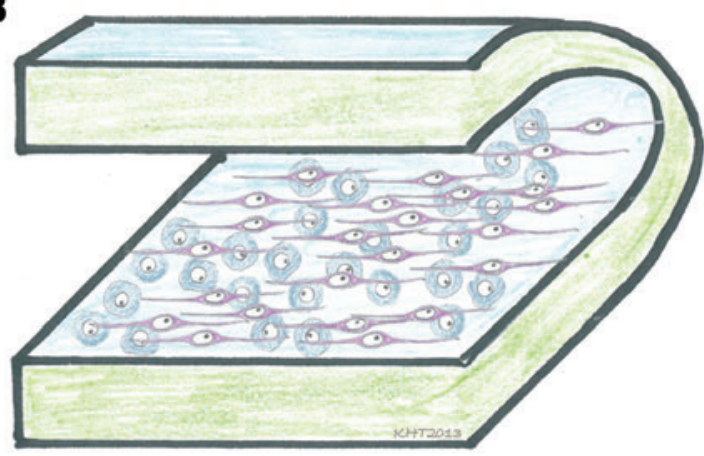

Bone marrow-derived mesenchymal stromal cells Schwann cells

FIG. 1. Illustration of a strategy for rolling chitosan films to become a hollow nerve guidance channels. (A) By tailoring the degree of acetylation (DA) when modifying the number of conjugated acetyl groups, the degradation rate of the films can be adjusted. (B) A chitosan film of adjusted DA further provides a valuable substrate for seeding aligned Schwann cells (SCs) and bone-marrow-derived mesenchymal stromal cells (BMSCs), both candidate cells for peripheral nerve tissue engineering. Color images available online at www.liebertpub.com/tea 
PAA Laboratories). The cell suspension was centrifuged for $5 \mathrm{~min}$ at $235 \mathrm{~g}$. Afterward cells were placed into a poly-Llysine (PLL)-coated (final concentration $0.5 \mathrm{ng} / \mathrm{mL}$; SigmaAldrich Chemie $\mathrm{GmbH}$ ) T25 culture flask (NUNC ${ }^{\mathrm{TM}}$ ) and incubated for $24 \mathrm{~h}$. On the next day half of the medium was replaced by fresh medium. About $1 \mathrm{mM}$ of arabinoside-c (Sigma-Aldrich Chemie $\mathrm{GmbH}$ ) was added for 2 days to suppress overgrowth by fibroblasts. Cells were then purified via immunopanning ${ }^{15}$ by magnetic beads Dynabeads ${ }^{\circledR}$ (Pan mouse IgG; Dynal Biotec ASA) coupled to anti-Thy-1 antibodies (own production from hybridoma cell line) and seeded into a new PLL-coated T25 culture flask. SC purification procedure was repeated once or twice until $>90 \%$ pure neoSC cultures were reached.

Adult rat SCs. Adult SCs (aSCs) were harvested from sciatic nerves of female Wistar rats (200 g, 8-16 weeks). In vitro predegeneration occurred for 14 days according to a protocol described previously by Haastert et al. ${ }^{14}$ Afterward primary cells were cultured in melanocyte growth medium (Promocell), $2 \mu \mathrm{M}$ Forskolin (Calbiochem), FGF-2 (10 ng/ $\mathrm{mL}$; PeproTech), bovine pituitary extract $(5 \mu \mathrm{g} / \mathrm{mL}$ BPE-26; Promocell), $10 \% \mathrm{FCS}$, and 1\% Pen/strep. After 1 week, highly enriched aSCs were obtained by using the magneticassisted cell sorting system MACS ${ }^{\circledR}$ (Miltenyi Biotec) following the manufacturer's protocol. Briefly, the unpurified cultures were detached and washed and the pellet was diluted in $998 \mu \mathrm{L}$ of MACS buffer prior to incubation with $2 \mu \mathrm{L}$ low-affinity nerve growth factor-receptor antibody (Millipore; AB 1554) for $15 \mathrm{~min}$ at $4^{\circ} \mathrm{C}$. After two washing steps, $40 \mu \mathrm{L}$ of microbead-conjugated anti-rabbit IgG was mixed with cell suspension in MACS buffer and incubated for further $15 \mathrm{~min}$ at $4^{\circ} \mathrm{C}$. After the incubation period, the cell suspension was flushed through the prepared MACS columns (Miltenyi; Size M) in the mini MACS magnet and the unbound fibroblasts were washed out. After removal from the magnet, bound aSCs were flushed with $2 \mathrm{~mL}$ MACS buffer. Highly purified aSCs were then seeded into poly-ornithin-laminin $^{14}$ (Sigma-Aldrich, Becton-Dickinson)coated T75 culture flasks.

Immortalized SCs. Rat immortalized SCs (iSCs) ${ }^{16}$ were cultured in DMEM supplemented with $10 \%$ FCS, $1 \%$ Pen/ strep, $1 \%$ sodium pyruvate, and $1 \%$ L-glutamine in noncoated culture flask.

Rat BMSCs. Isolation and culturing of rat BMSCs from the femur bone marrow were performed as described before. ${ }^{17}$ Isolated cells were cultured in alpha-MEM supplemented with $15 \%$ fetal bovine serum (FBS; Invitrogen) and $1 \%$ antibiotic/antimycotic $\left(\right.$ Sigma $\left.^{\circledR}\right)$ in sterile T75 and T175 culture flasks until confluence was reached. Cells at passages 4-6 were detached, seeded on top of the chitosan films, and cultured with alpha-MEM with $10 \%$ FBS and $1 \%$ antibiotic/antimycotic.

\section{Cell seeding on chitosan films}

Rat neoSCs (passage 6-8) and iSCs were seeded into 24well plates with a density of $1 \times 10^{4}$ cells/well (iSCs) or $3.5 \times 10^{4}$ cells/well (neoSCs) on PLL (Sigma)-coated wells or noncoated chitosan films clamped into cell crowns
(Scaffdex $\left.^{(}\right)$in a volume of $400 \mu \mathrm{L}$ of cell-specific culture medium. After $1 \mathrm{~h}$ of incubation, more medium was added to a total volume of $900 \mu \mathrm{L} /$ well. The medium was changed twice a week and cells were cultivated over 5 days in vitro (DIV) for iSCs and 7 DIV for neoSCs. Rat aSCs (passage 36) and rat BMSCs (passage 4-6) were seeded on coated coverslips into 24-well plates with a density of either $3.5 \times 10^{4}$ cells/well (aSCs, poly-ornithin laminin coating) or $2 \times 10^{4}$ cells/well (BMSCs, poly-D-lysine; Sigma) or on noncoated chitosan films clamped into cell crowns (Scaffdex) in a volume of $75 \mu \mathrm{L}$ of cell-specific culture medium. After $1 \mathrm{~h}$ of incubation, more medium was added to a total volume of $800 \mu \mathrm{L} /$ well. Medium was changed twice a week and cells were cultivated until 7 DIV.

\section{Metabolic activity evaluation by WST-1 and MTS assays}

WST-1 test or MTS assay was chosen to evaluate metabolic activity of different cell types seeded on chitosan films. The respective assay was chosen with regard to the best experiences from previous studies with either test for the cell types to be analyzed.

For the iSC and neoSC experiments, cell metabolic activity was compared in the WST-1 test (Cell Proliferation Reagent; Roche). WST-1 \{4-[3-(4-Iodophenyl)-2(4nitrophenyl)-2H-5-tetrazolio]-1,3-benzene disulfonate $\}$ is reduced by dehydrogenase enzymes to a dark red compound in cells with metabolic activity, and is particularly suitable for the cell types used. After cultivation periods-1, 3, and 5 DIV for iSCs or 3, 5, and 7 DIV for neoSCs - cell crowns were transferred to new wells of the same plate and cellspecific culture medium containing the WST-1 compound was added (1:10, $400 \mu \mathrm{L} /$ controls and $900 \mu \mathrm{L} /$ cell crown). Cells were incubated for $\sim 3.5 \mathrm{~h}$ at $37^{\circ} \mathrm{C}$ in humidified atmosphere with $8 \%(\mathrm{v} / \mathrm{v}) \mathrm{CO}_{2}$. Afterward triplicates of $100 \mu \mathrm{L}$ from each sample were transferred to 96-well plates and the optical density (OD) was measured at $450 \mathrm{~nm}$ using a multiwell plate reader $\left(\mathrm{EL}_{\mathrm{x}} 800\right.$ BioTek Instruments). The test was performed in $n=3$ experiments with each two sister cultures per condition.

For aSCs and BMSCs, cell metabolic activity was compared in the MTS test (Cell Titer $96{ }^{\circledR} \mathrm{AQ}$ ueous Cell Proliferation Assay; Promega). The MTS compound [3-(4,5 dimethylthiazol-2-yl)-5-(3-carboxymethoxy-phenyl)-2 (4 sulfophenyl) $2 \mathrm{H}$-tetrazolium inner salt] is reduced by dehydrogenase enzymes to a brownish formazan product in cells with metabolic activity. After 2 and 7 DIV, coverslips and cell crowns were transferred to new wells of the same plate and serum-free culture medium (aSCs) or DMEM (BMSCs) containing the MTS compound was added (5:1, $350 \mu \mathrm{L} /$ controls and $850 \mu \mathrm{L} /$ cell crown). Cells were incubated for $\sim 3 \mathrm{~h}$ at $37^{\circ} \mathrm{C}$ in humidified atmosphere with $5 \%$ (v/v) $\mathrm{CO}_{2}$. Afterward triplicates of $100 \mu \mathrm{L}$ from each sample were taken to 96-well plates and the OD was measured at $490 \mathrm{~nm}$ using a multiwell plate reader (Biorad Model 680). This test was performed in $n=3$ experiments with each three sister cultures per condition.

\section{Cell proliferation}

The cell proliferation rate of aSCs and BMSCs was determined with the BrdU colorimetric assay (Roche) that is 
based on the detection of BrdU (thymidine analogue) incorporation into genomic DNA of proliferating cells by binding of anti-BrdU ( $\alpha$-BrdU) antibody. The procedure was assessed according to the manufacturing protocol. Therefore at 2 and 7 DIV, cell-seeded coverslips and chitosan films in cell crowns were incubated with $10 \mu \mathrm{M}$ BrdU diluted in cellspecific culture medium for $24 \mathrm{~h}$, afterward fixed in FixDenat solution and then incubated for $\sim 1.5 \mathrm{~h}$ in a solution containing $\alpha$-BrdU-POD. After washing $(3 \times$ in washing buffer) and 15-min incubation with TMB solution, triplicates of $100 \mu \mathrm{L}$ of each condition were transferred to 96well plates and the OD was measured in an ELISA plate reader (BioRad Model 680) at 405/490 nm. Cell proliferation was determined in $n=3$ experiments with each three sister cultures per condition.

\section{Coculture experiments of aSCs with rat BMSCs}

To evaluate stimulatory or inhibitory effects on the growing characteristics and the proliferation behavior of cocultured aSCs $\left(1.75 \times 10^{4}\right.$ cells/well $)$ and BMSCs $\left(1 \times 10^{4}\right.$ cells/well), a direct coculture on chitosan films and polyD-lysine-coated glass coverslips was performed. Cells were grown in aSC medium over 2 and 7 DIV in $n=3$ experiments with each three sister cultures per condition.

\section{Preparation and cultivation of primary dissociated rat dorsal root ganglia}

Dorsal root ganglia (DRGs) were dissected from neonatal Wistar rats (P1-P3) and collected in HBSS medium (Hank's balanced salt solution without magnesium and calcium; PAA Laboratories). After isolation, ganglia were incubated in dissociation solution (HBSS, trypsin-0.125\% [Gibco], and $0.1 \%$ DNase [0.5\% stock; Roche Diagnostics]) for $20 \mathrm{~min}$ at $37^{\circ} \mathrm{C}$. Then, collagenase IV (160 U/mg; PAA Laboratories) was added for another $25 \mathrm{~min}$. Digestion of DRGs was stopped by adding N2 medium with 3\% FCS (DMEM-F12 [PAA Laboratories], $1 \times$ N2-supplement [100×; Gibco], 0.25\% bovine serum albumin [BSA, fraction V, 25\% stock; SigmaAldrich Chemie GmbH], 2 mM L-glutamine, 1\% Pen/strep, and $1 \mathrm{mM}$ sodium pyruvate [PAA Laboratories]). Ganglia were then mechanically dissociated in a homogenous suspension using a fire-polished glass Pasteur pipette. After centrifugation, $10 \times 10^{4} \mathrm{DRG}$ cells were seeded on top of poly-ornithin-laminin-coated coverslips (control condition) or plain or cell-seeded chitosan films, respectively.

After $24 \mathrm{~h}$, control condition cultures were separated into nontreated and $50 \mathrm{ng} / \mathrm{mL}$ free recombinant nerve growth factor (NGF, Invitrogen)-supplemented sister cultures. All other cultures were kept without NGF supplement. Cells were grown over 2 and 7 DIV in sister cultures.

\section{Immunocytochemistry and phalloidin/DAPI staining}

To evaluate cell morphologies immunocytochemistry was performed. Therefore, coverslips or chitosan films were gently washed with phosphate-buffered saline (PBS) and fixed for $30 \mathrm{~min}$ with $4 \%$ paraformaldehyde (Merck). Rat aSCs were incubated overnight $\left(4^{\circ} \mathrm{C}\right)$ with SC-specific $\alpha$ S100 antibody (polyclonal, 1:200; Dako) in PBS/0.3\% Triton $\mathrm{X}-100 / 5 \%$ BSA (PAA) solution. After washing with
PBS, an incubation with Cy2-labeled goat $\alpha$-rabbit secondary antibody (1:2000; Invitrogen) for $\sim 1.5 \mathrm{~h}$ at RT followed. To identify BMSCs, their actin filaments were stained for $30 \mathrm{~min}$ with phalloidin-tritc fluorescence stain (1:500; Sigma). All cell nuclei were counterstained with DAPI (1:1000; Invitrogen D1306). After final washing, coverslips and chitosan films were mounted on glass slides with Shandon-Immu-Mount (Thermo Fisher ${ }^{\circledR}$ ).

To distinguish between the cell populations in coculture experiments, cells were fixed and stained by Phalloidin/ DAPI for $30 \mathrm{~min}$ and then incubated overnight with the $\alpha$ S100 antibodies followed by their detection with Cy2labeled goat $\alpha$-rabbit secondary antibody. Microscopical analyses were carried out under the fluorescence microscope (BX61; Olympus). For quantification, six images per condition were captured at $\times 20$ magnification using Cell $\mathrm{P}^{\circledR}$ (Olympus) and Image $\mathrm{J}$ software (Wayne Rasband).

For detection of neurite outgrowth, dissociated DRG cultures on coated coverslips and chitosan films were washed and fixed on 2 and 7 DIV as described previously for aSCs. Blocking of unspecific antibody binding was induced by incubation with PBS/0.3 Triton X-100 containing 3\% normal goat serum (NGS, Gibco) for $1 \mathrm{~h}$ at RT. Dissociated DRGs were incubated overnight $\left(4^{\circ} \mathrm{C}\right)$ with neuron-specific $\alpha$ - $\beta$-III-tubulin antibody (monoclonal, 1:500; Upstate Biotechnology) in PBS/0.3\% Triton X-100 containing $1 \%$ NGS. After washing with PBS, incubation with Alexa 647-labeled goat $\alpha$-mouse secondary antibody (1:400; Invitrogen) for $\sim 1.5 \mathrm{~h}$ (RT) followed. Cell nuclei were counterstained with DAPI (1:1000; Sigma-Aldrich). After final washing, coverslips and chitosan films were mounted and coverslipped as described previously.

\section{Statistics}

Statistical analysis was performed using GraphPad Prism software version 5.0.3.0 for windows (GraphPad Software, Inc.). Data represent mean \pm standard error of the mean (SEM). Statistical differences were assessed by two-way ANOVA followed by Bonferroni post hoc test for multiple comparisons. $p$-Values with $p<0.05$ were regarded as statistically significant.

\section{Results}

The mechanical properties of the chitosan films were determined as listed in Table 1. Thickness, tensile strength, and Young's modulus were not significantly affected by beta-sterilization.

The scanning electron microscopic images (Fig. 2A) acquired revealed a flat and smooth surface of the chitosan films with minor defects. Additionally, from the FTIR analysis (Fig. 2B), a typical spectrum of chitosan, confirming its saccharide structure, ${ }^{18}$ was obtained; namely,

A band at $3441 \mathrm{~cm}^{-1}$ corresponds to the combined peaks of the $\mathrm{NH}_{2}$ and $\mathrm{OH}$ group stretching vibration of chitosan.

The bands at 1641 and $1380 \mathrm{~cm}^{-1}$ correspond to the $\mathrm{C}=\mathrm{O}$ and $\mathrm{C}-\mathrm{O}$ stretching of amide group.

A band at wavenumber $1598 \mathrm{~cm}^{-1}$ is due to the $\mathrm{N}-\mathrm{H}$ deformation of amino groups.

Wavenumbers 1156 and $1025 \mathrm{~cm}^{-1}$ correspond to the symmetric stretching of the C-O-C. 
Table 1. Results of the Mechanical Property Testing of Nonsterile Chitosan Films and Chitosan Films Sterilized with Beta-Radiation

\begin{tabular}{llccc}
\hline Sample & Sterile & $\begin{array}{c}\text { Thickness } \\
(\mathrm{mm})\end{array}$ & $\begin{array}{c}\text { Tensile strength } \\
\text { straight pull }(\mathrm{N})\end{array}$ & $\begin{array}{c}\text { Young's } \\
\text { modulus }(\mathrm{MPa})\end{array}$ \\
\hline W3R3-1205/01 & Yes & $0.04 \pm 0.011$ & $5.6 \pm 2.2$ & $0.47 \pm 0.09$ \\
W3R3-1205/01 & No & $0.035 \pm 0.005$ & $6.8 \pm 1.69$ & $0.41 \pm 0.15$ \\
\hline
\end{tabular}

Data are presented as mean \pm standard deviation.

\section{Metabolic activity of different primary and iSCs increased over culture periods on chitosan films}

To investigate the initial cytocompatibility properties of chitosan films, direct contact assays with rat iSCs and neoSCs were performed and compared with control cultures on PLL coating. The interaction of the cells with the substrates was evaluated after different culture periods by measuring their metabolic activity in the WST-1 assay. For both cell types metabolic activity was, at all time points investigated, significantly higher in control cultures than on noncoated chitosan films. However, cells cultured on chitosan films were viable and showed a significant increase in their metabolic activity over time as did the control cultures (Fig. 3A, B).

\section{aSCs and BMSCs show differences in metabolic activity and proliferation behavior on chitosan film substrates}

For both cell types metabolic activity was, at all time points investigated, significantly higher in control cultures than on noncoated chitosan films and it showed an increase over time (Fig. 3C, D). In contrast to what was seen with iSCs and neoSCs, rat aSCs showed a nonsignificant decrease of their metabolic activity (MTS assay) between 2 and 7 DIV (Fig. 3C). Rat BMSCs however demonstrated the opposite, an increasing metabolic activity from 2 to 7 DIV (Fig. 3D).
Interestingly, also the proliferation behavior of rat aSCs and BMSCs showed opposite characteristics on chitosan film substrates (Fig. 3E, F). During the measured period of $24 \mathrm{~h}$ from 2 to 3 DIV nonsignificantly less aSCs were proliferating than during $24 \mathrm{~h}$ between 7 and 8 DIV. In the latter time period the proliferation rate was significantly higher in chitosan film cultures than in controls (Fig. 3E). In contrast, nonsignificantly higher proliferation rates were detected in early chitosan film BMSC cultures (2-3 DIV) than in older ones (7-8 DIV) with no significant difference to control cultures (Fig. 3F).

The viability of aSCs and BMSCs on chitosan film substrates was confirmed by immunofluorescence (Fig. 4), showing aSCs with their typical spindle-shaped morphology, while BMSCs appeared on 2 DIV with a contracted morphology that changed to their regular more flattened morphology only at 7 DIV.

\section{Relative cell numbers of BMSCs and aSCs were stable when cocultured on chitosan film substrates}

A direct coculture of aSCs and BMSCs on chitosan films and control substrates revealed in immunofluorescence that aSCs grew on top of the BMSCs (Fig. 5A, B). The topography of cell growth indicates that BMSCs served as a feeder layer for aSCs in the coculture system. The number of viable cells was determined in order to calculate the relative proportion of the two cell populations when cultured on the
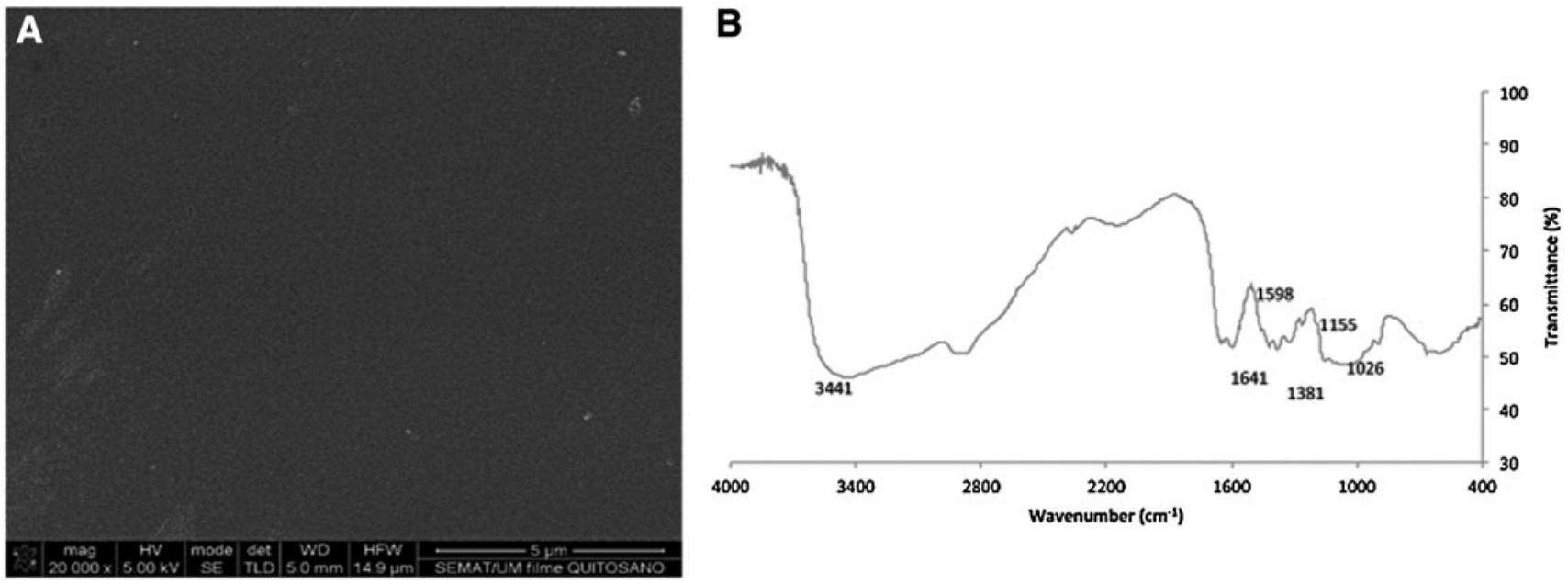

FIG. 2. Characterization of chitosan films. Scanning electron microscopy (A) analysis revealed a relative smooth surface while with Fourier transform infrared spectroscopy spectra (B) a typical spectrum of chitosan was obtained. 

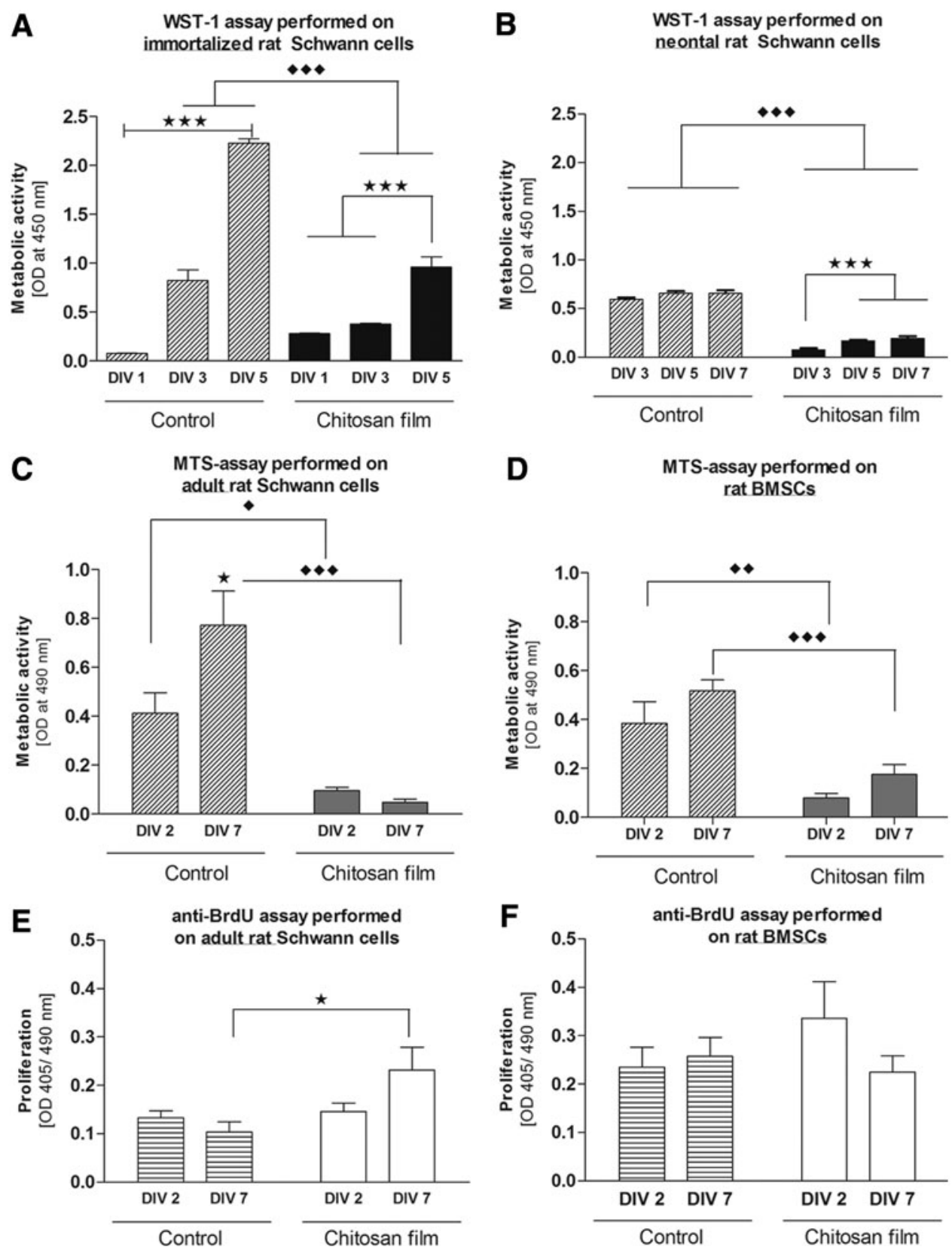

FIG. 3. Cell metabolic activity of different cell types on chitosan films. (A) Rat immortalized SCs (iSCs; $1 \times 10^{4}$ cells $/$ well) and (B) rat neonatal SCs (neoSCs; $3.5 \times 10^{4}$ cells/well) were cultured on different chitosan films (5\% DA) for up to 7 days in vitro (DIV). The metabolic activity measured in the WST-1 assay (optical density [OD] $450 \mathrm{~nm}$ ) showed an increase after 3 DIV for both iSCs and neoSCs. Control condition cultures always displayed significantly higher metabolic activities than experimental cultures. Bars represent mean \pm standard error of the mean (SEM) $(n=3$ experiments, $* * * p<0.001)$. Cell metabolic activity of rat adult SCs (aSCs) and rat BMSCs cultured on chitosan films. (C) aSCs and (D) BMSCs were cultured on chitosan films for 2 and 7 DIV. Metabolic activity showed a nonsignificant decrease after 7 DIV in aSCs while in BMSCs it is increased. Control condition cultures always displayed significantly higher metabolic activities than experimental cultures. Bars represent mean \pm SEM ( $n=3$, two-way ANOVA $\diamond \bullet<0.0001, \diamond p<0.001, \bullet p<0.05)$. Proliferation rates of rat aSCs and BMSCs when cultured on chitosan films for different periods. (E) aSCs and (F) BMSCs were cultured for 2 and 7 DIV. Proliferation rate was determined for a 24-h window preceding fixation on 2 and 7 DIV. aSCs showed a nonsignificant increase in proliferation with prolonged culture, while BMSCs showed a nonsignificant decrease that was not significant. Bars represent mean \pm SEM $(n=3$; two-way ANOVA * $p<0.05)$.

substrates (Fig. 5C). While the relative number of cultured BMSCs increased significantly on control substrates from $\sim 61 \%$ at 2 DIV to $\sim 75 \%$, the relative number of aSCs decreased significantly from $\sim 40 \%$ to $\sim 25 \%$. On chitosan film substrates the change in relative cell numbers is nego- tiable for both cell types and similar to control conditions at 7 DIV. The relative proportion in early cultures on chitosan films is however significantly different to cultures on control substrates, with higher relative numbers of BMSCs and less relative numbers of aSCs growing on the chitosan films. 

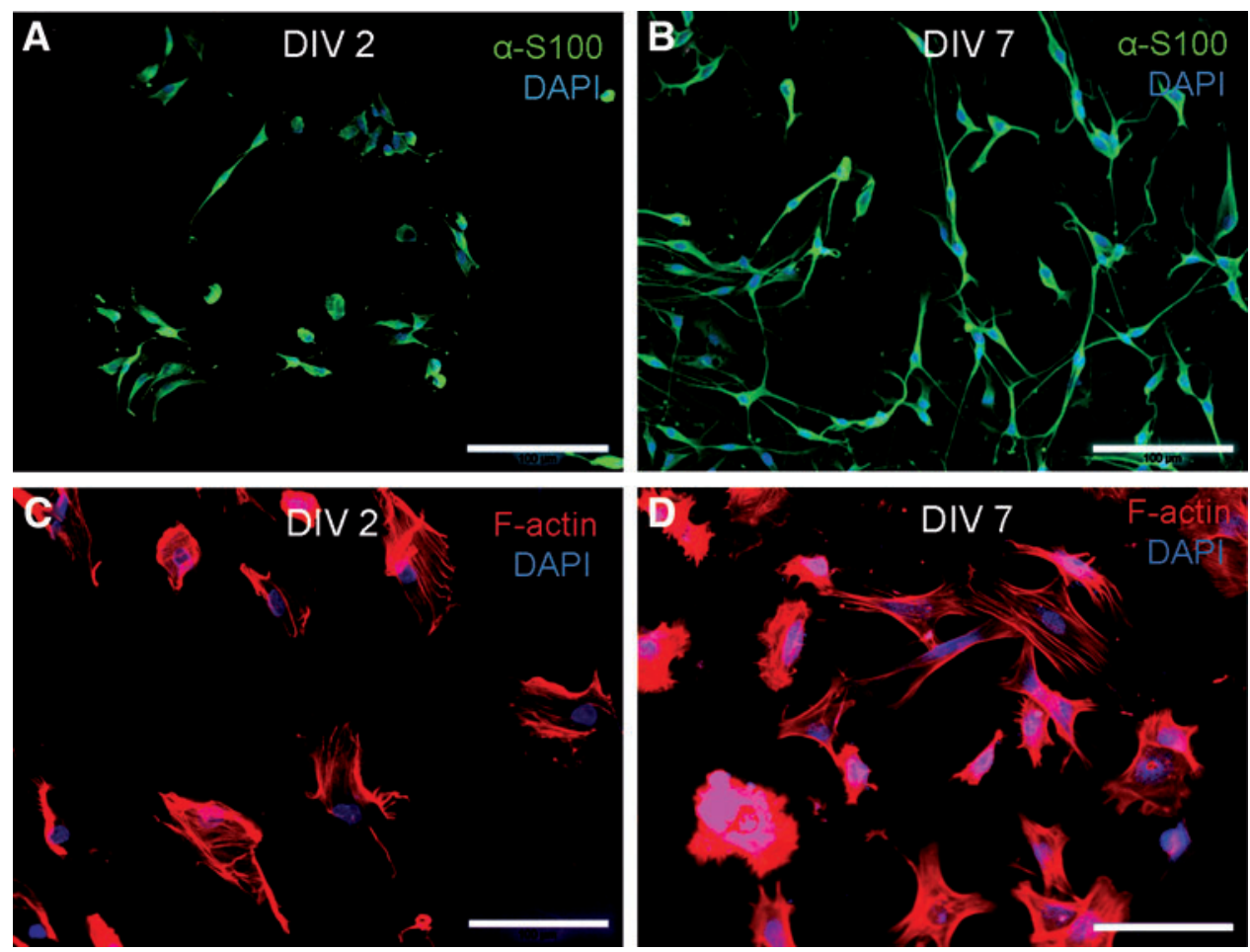

FIG. 4. Immuno-

fluorescence of rat aSCs and BMSCs on chitosan films. aSCs (green, $\alpha$-S100) were viable and showed their typical spindle-shaped morphology on 2 DIV (A) and 7 DIV (B). BMSCs (red, Factin filaments) were viable but showed a contracted morphology on 2 DIV (C) and presented their typical fibroblast-like morphology only on 7 DIV (D). All nuclei counterstained with DAPI. Scale bar $=100 \mu \mathrm{m}$. Color images available online at www.liebertpub.com/tea
Axonal outgrowth on chitosan films is increased after preseeding with aSC-BMSC cocultures

To proof the principle that cocultures of aSCs and BMSCs could support neurite outgrowth through chitosan- film-based nerve grafts, dissociated DRG cells were cultured on poly-ornithin-laminin-coated controls or on top of either plain films or those seeded with aSC-BMSC cocultures 2 and $7 \mathrm{DIV}$, respectively. Under control conditions less neurite outgrowth was detected (Fig. 6A, B) compared with
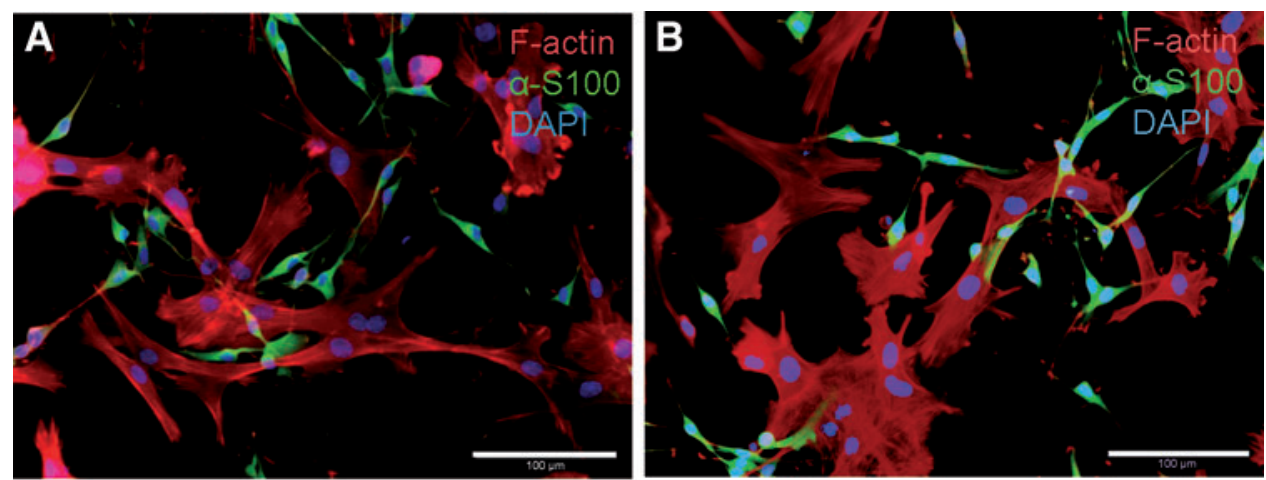

Co-culture of
C rat BMSCs and adult rat Sch

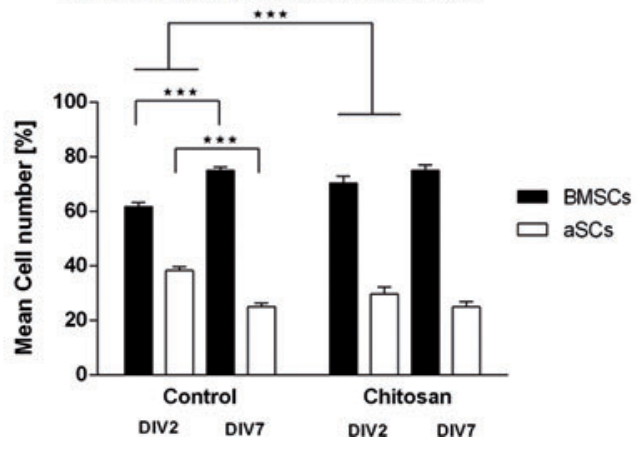

FIG. 5. Quantification of relative cell numbers $(\%)$ of BMSCs and aSCs in direct coculture on chitosan films versus control substrates. Significant differences between 2 and 7 DIV were observed on control substrates for both BMSCs and aSCs, but in opposite direction. No significant differences in relative cell numbers were detected for either cell type over time in cocultures on chitosan films (C). Bars shown as mean \pm SEM; $n=3$. Representative photomicrographs of BMSCs stained for F-actin filaments (phalloidin in red) and $\alpha$-S100-positive aSCs (green) on 2 DIV (A) and 7 DIV (B). Scale bar $=$ $100 \mu \mathrm{m}$. *** $p<0.0001$. Color images available online at www.liebertpub .com/tea 
FIG. 6. Immuno-

fluorescence of rat primary dissociated dorsal root ganglia (DRGs) axonal outgrowth. Dissociated DRG cells (red, $\alpha$ - $\beta$-III-tubulin) in absence of nerve growth factor (NGF) (control 1) on 2 DIV (A) and a slightly increased neurite outgrowth on 7 DIV (B). In presence of NGF (control 2) on 2 DIV (C) with an increased neurite outgrowth on 7 DIV (D). On plain chitosan films cluster formation with increased neurite outgrowth occurred till 2 DIV (E) and was further increased till 7 DIV (F). (G, H) On chitosan films preseeded with cocultures of aSCs and BMSCs, axonal outgrowth and network formation was further intensified over time. Blue: nuclear staining (DAPI); scale bars = $200 \mu \mathrm{m}$. Color images available online at www .liebertpub.com/tea
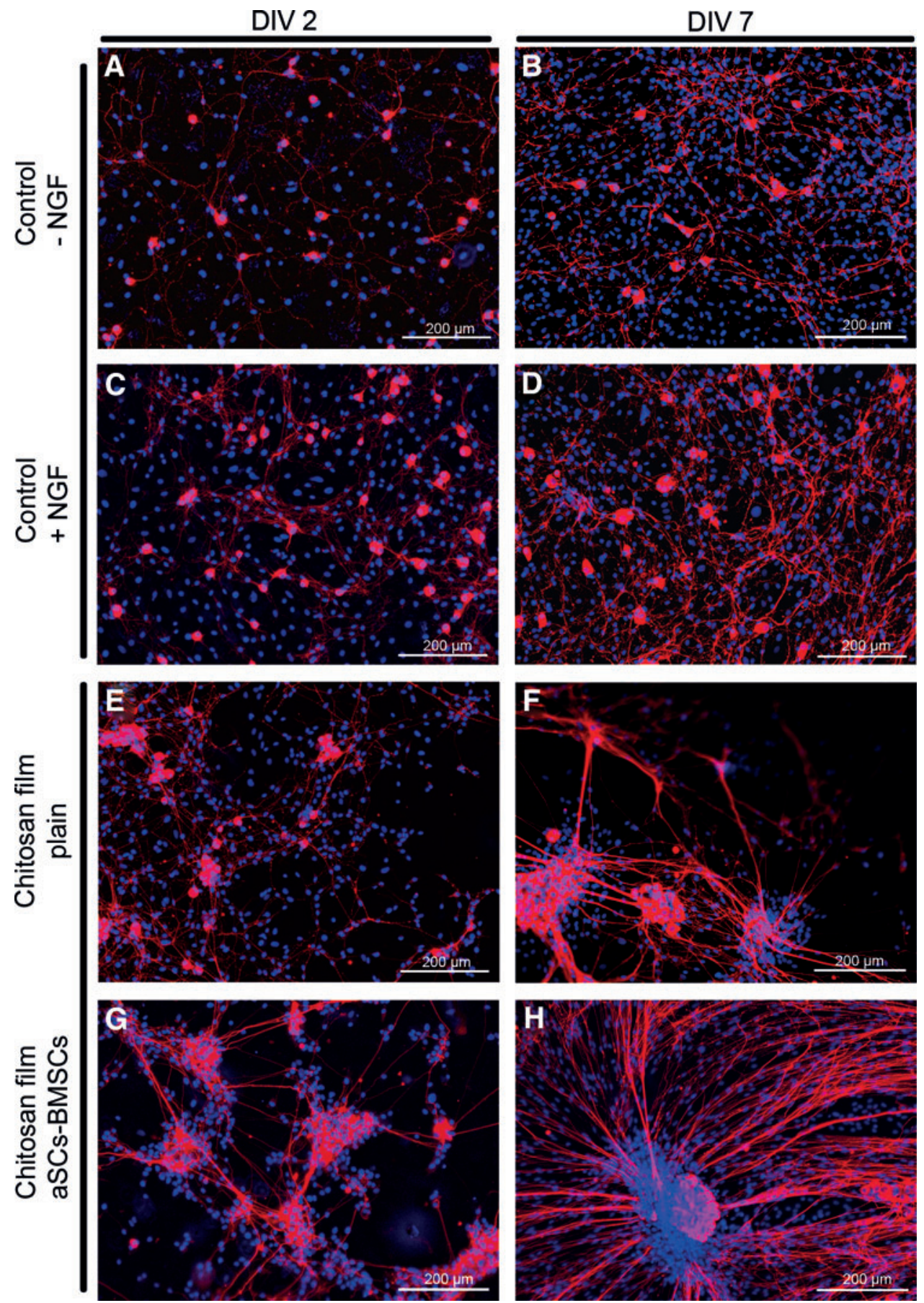

control culture supplemented with NGF (Fig. 6C, D). On plain chitosan films, without NGF supplementation, some clustering of the dissociated DRG cells was visible (Fig. 6E, F) but neurite outgrowth was supported to a higher extend than under control conditions plus NGF. The neurite outgrowth was however best supported when dissociated DRG cells were seeded on aSC-BMSC-coculture-preseeded chitosan films (no NGF added, Fig. 6G, H).

\section{Discussion}

This study was performed with the prospect of a novel entubulation strategy in which chitosan films could be see- ded with supportive cells and used as a composite guiding conduit for peripheral nerve repair. We characterized chitosan films with a DA of $\sim 5 \%$ in terms of mechanical properties and surface topography. The degradation rates of chitosan films of different DA were determined in another study before and chitosan films with a DA of $\sim 5 \%$ demonstrated a mass loss $<10 \%$ over 28 days in presence of lysozyme in vitro. ${ }^{18} \mathrm{We}$ also evaluated the biocompatibility of the chitosan films with different cell types.

Scanning electron microscopic analysis of the chitosan films revealed a flat and smooth surface. It is known that surface properties of biomaterials, such as their chemical structure and topography, are essential for adhesion and 
proliferation of cells ${ }^{19}$ and in that context important for acceptance or rejection of those materials in vivo. The monitored surface as such showed no negative properties that would limit the use of chitosan films in peripheral nerve repair. Biocompatibility of chitosan materials in terms of better cell adhesion and proliferation can be modified by deacetylation of the raw material. ${ }^{18}$ Cell lines are widely used for in vitro cytotoxicity assessments but the transmission to a clinical situation is restricted to data obtained with primary target cells or data obtained in vivo. To gain a more comprehensive view on the chitosan film properties, beside iSCs, primary neoSCs and aSCs, with a special focus on the aSCs, were analyzed. SCs are the main players in the scenario of peripheral nerve regeneration. In a clinical setting of peripheral nerve reconstruction aSCs are crucially involved when focusing on the mean age of the population with peripheral nerve injuries. ${ }^{20,21}$ As a second cell type with regeneration-promoting properties, ${ }^{22-24}$ BMSCs were analyzed.

All cell populations were grown on chitosan films with $5 \%$ DA that has been considered to be optimal for glial cells, given that previous studies demonstrated adverse effects for different cell types when higher DAs were used. ${ }^{18,25-27}$ The results of the direct-contact assays clearly demonstrated that our chitosan films provide a suitable biomaterial with good cell adhesion and proliferation properties and allow axonal outgrowth.

iSCs and primary neoSCs as well as BMSCs demonstrated an increased metabolic activity over culture time on chitosan films (Fig. 3A, B, and D). This is in agreement with the metabolic activity changes found for other cell types from different origin when seeded on chitosan with DA $<10 \%{ }^{18,26,28,29}$ In contrast, aSCs cultured on the chitosan films showed a nonsignificant decrease in their metabolic activity on 7 DIV (Fig. 3C). These cell-type-specific differences are of particular importance with respect to the impact of aSCs on the peripheral nerve regeneration process. Although other authors conclude from a reduced metabolic activity to a reduced cell viability, ${ }^{25,28}$ we did not see impaired cell viability when analyzing our cultures in immunofluorescence. The significantly higher metabolic activity measured under control conditions has to be attributed to the highly optimized culture conditions induced by the cell-specific coating used. From the chitosan film cultures, however, we assume that aSCs adapt faster to the biomaterial with a reduced reaction to the chitosan and a subsequently reduced metabolism than iSCs or neoSCs or BMSCs. Also, the significant increase of proliferating aSCs until 7 DIV (Fig. 3E) argues against a reduced viability of this cell type on chitosan films. With regard to cell proliferation, BMSCs again demonstrated an opposite behavior to aSCs. Proliferation rates at 2 DIV were lower in aSCs than in BMSCs and at 7 DIV the opposite was detected. These observations can be explained by a reduction of cell-free material surface needed for further cell expansion due to increased cell numbers until 7 DIV for BMSCs. ${ }^{30}$ aSCs showed a slower growth and could therefore still increase their proliferation rates at 7 DIV. Despite of cell-typespecific differences regarding metabolic activity and proliferation behavior between aSCs and BMSCs, both cell types were viable and adhered well to the substrate. These observations were also confirmed by immunofluorescence showing typical spindle-shaped aSCs on 2 and 7 DIV (Fig. 4A, B) while BMSCs appeared initially contracted but with prolonged culture they changed to their regular morphology (Fig. 4C, D). The latter is another evidence for the conclusion that BMSCs need more time to adapt to the chitosan surface than aSCs.

Recent investigations demonstrated that transplanted mesenchymal stromal cells are therapeutically efficient in models of different neurological disorders, including peripheral nerve regeneration, either by differentiation into an SC-like phenotype or by using them as a source of growth factors. ${ }^{31-33}$ To study the possible synergistic or inhibitory effects of aSCs and BMSCs, both cell types were directly cocultured on chitosan films (Fig. 5). Similar to the results obtained from monocultures both cell types were viable and adhered to the chitosan substrate. It is noteworthy that aSCs grew on top of the BMSCs, using them as a feeder layer. In prospect of composite nerve entubulation strategies, the demonstrated growth behavior would allow the aSCs to be in direct contact with regrowing axons while receiving trophic support from the underlying BMSCs. To detect cell type interactions, relative cell numbers of both cell populations were determined under control conditions and on chitosan films. While under control conditions, the relative cell number of BMSCs increased on 7 DIV and that of aSCs decreased; on the chitosan films, the relative cell number for both aSCs and BMSCs remained stable. This indicates that aSCs and BMSCs did not negatively affect each other. Further, axonal outgrowth from dissociated DRG cells cultured on chitosan films was evidently stronger than under positive control conditions (poly-ornithin-laminin-coated glass coverslips and NGF supplementation, Fig. 6A-F). Chitosan films preseeded with aSC-BMSC cocultures further proved to be even more supportive of axonal outgrowth (Fig. 6G, H).

In future studies, the coculture of aSCs and BMSCs needs to be further analyzed for the effects of the BMSC secretome on aSC behavior. Therefore higher cell densities and prolonged culture periods need to be established.

We demonstrate here as a proof-of-principle that chitosan films of 5\% DA are a suitable biomaterial that supports viability and adhesion of glial cells, specifically SCs and BMSCs, and that both cell types can be cocultured in order to cotransplant them for peripheral nerve reconstruction. This conclusion is supported by the finding that massive neurite outgrowth from sensory neurons is induced on chitosan films preseeded with aSC-BMSC cocultures. A composite entubulation strategy including chitosan films seeded with autologous SCs and BMSCs could not only be supportive for axonal regrowth but also for the growth and function of resident SCs in the adjacent nerve ends. These cells could also be positively influenced by the secretome of the transplanted BMSCs.

\section{Acknowledgments}

This work was supported by the European Community's Seventh Framework Programme (FP7-HEALTH-2011) under grant agreement No. 278612. This work was also cofunded by Programa Operacional Regional do Norte (ON.2-O Novo Norte), ao abrigo do Quadro de Referência Estratégico Nacional (QREN), and através do Fundo Europeu de 
Desenvolvimento Regional (FEDER). The authors gratefully acknowledge the delivery of the chitosan raw material by Altakitin S.A., Portugal, and the fabrication of chitosan films by Medovent $\mathrm{GmbH}$, Germany.

\section{Disclosure Statement}

No competing financial interests exist.

\section{References}

1. Ciardelli, G., and Chiono, V. Materials for peripheral nerve regeneration. Macromol Biosci 6, 13, 2006.

2. Millesi, H. Bridging defects: autologous nerve grafts. Acta Neurochir Suppl 100, 37, 2007.

3. Deumens, R., Bozkurt, A., Meek, M.F., Marcus, M.A.E., Joosten, E.A.J., Weis, J., et al. Repairing injured peripheral nerves: bridging the gap. Prog Neurobiol 92, 245, 2010.

4. Ray, W.Z., and Mackinnon, S.E. Management of nerve gaps: autografts, allografts, nerve transfers, and end-to-side neurorrhaphy. Exp Neurol 223, 77, 2010.

5. Lundborg, G. Alternatives to autologous nerve grafts. Handchir, Mikrochir, Plast-Chir 36, 1, 2004.

6. Mackinnon, S.E., and Hudson, A.R. Clinical application of peripheral nerve transplantation. Plast Reconstruct Surg 90, 695, 1992.

7. Haastert-Talini, K., Geuna, S., Dahlin, L.B., Meyer, C., Stenberg, L., Freier, T., Heimann, C., Barwig, C., Pinto, L.F.V., Raimondo, S., Gambarotta, G., Ribeiro-Samy, S., Sousa, N., Salgado, A.J., Ratzka, A., Wrobel, S., and Grothe, C. Chitosan tubes of varying degrees of acetylation for bridging peripheral nerve defects. Biomaterials 34, 9886, 2013.

8. Freier, T., Hamann, M.J., Katayama, Y., Musoke-Zawedde, P., Piotrowicz, A., and Yuan, Y.S.M. Biodegradable polymers in neural tissue engineering. In: Mallapragada, S.N.B., ed. Biodegradable Polymeric Materials and Their Applications. American Scientific Publishers, California, USA, 2005, pp. 1-49.

9. Muir, D. The potentiation of peripheral nerve sheaths in regeneration and repair. Exp Neurol 223, 102, 2010.

10. Keilhoff, G., Goihl, A., Langnäse, K., Fansa, H., and Wolf, G. Transdifferentiation of mesenchymal stem cells into Schwann cell-like myelinating cells. Eur J Cell Biol 85, 11, 2006.

11. Keilhoff, G., Goihl, A., Stang, F., Wolf, G., and Fansa, H. Peripheral nerve tissue engineering: autologous Schwann cells vs. transdifferentiated mesenchymal stem cells. Tissue Eng 12, 1451, 2006.

12. Teixeira, F.G., Carvalho, M.M., Sousa, N., and Salgado, A.J. Mesenchymal stem cells secretome: a new paradigm for central nervous system regeneration? Cell Mol Life Sci 70, 3871, 2013.

13. Chatelet, C., Damour, O., and Domard, A. Influence of the degree of acetylation on some biological properties of chitosan films. Biomaterials 22, 261, 2001.

14. Haastert, K., Mauritz, C., Chaturvedi, S., and Grothe, C. Human and rat adult Schwann cell cultures: fast and efficient enrichment and highly effective non-viral transfection protocol. Nat Protoc 2, 99, 2007.

15. Haastert, K., Grosskreutz, J., Jaeckel, M., Laderer, C., Bufler, J., Grothe, C., et al. Rat embryonic motoneurons in long-term co-culture with Schwann cells-a system to in- vestigate motoneuron diseases on a cellular level in vitro. $\mathrm{J}$ Neurosci Methods 142, 275, 2005.

16. Grothe, C., Meisinger, C., Holzschuh, J., Wewetzer, K., and Cattini, P. Over-expression of the $18 \mathrm{kD}$ and $21 / 23 \mathrm{kD}$ fibroblast growth factor-2 isoforms in PC12 cells and Schwann cells results in altered cell morphology and growth. Brain research. Mol Brain Res [Internet] 57, 97, 1998.

17. Salgado, A.J., Gomes, M.E., Coutinho, O.P., and Reis, R.L. Isolation and osteogenic differentiation of bone-marrow progenitor cells for application in tissue engineering. Methods Mol Biol (Clifton, N.J.) 238, 12, 2004.

18. Freier, T., Koh, H.S., Kazazian, K., and Shoichet, M.S. Controlling cell adhesion and degradation of chitosan films by N-acetylation. Biomaterials 26, 5872, 2005.

19. Chang, H.-I., and Wang, Y. Cell Responses to Surface and Architecture of Tissue Engineering Scaffolds in Regenerative Medicine and Tissue Engineering-Cells and Biomaterials. Eberli, D., ed. InTech; Europe, 2011.

20. Asplund, M., Nilsson, M., Jacobsson, A., and von Holst, H. Incidence of traumatic peripheral nerve injuries and amputations in Sweden between 1998 and 2006. Neuroepidemiology 32, 217, 2009.

21. Noble, J., Munro, C.A., Prasad, V.S., and Midha, R. Analysis of upper and lower extremity peripheral nerve injuries in a population of patients with multiple injuries. J Trauma 45, 116, 1998.

22. Uccelli, A., Benvenuto, F., Laroni, A., and Giunti, D. Neuroprotective features of mesenchymal stem cells. Best practice \& research. Clin Haematol 24, 59, 2011.

23. Ribeiro, C.A., Salgado, A.J., Fraga, J.S., Silva, N.A., Reis, R.L., and Sousa, N. The secretome of bone marrow mesenchymal stem cells-conditioned media varies with time and drives a distinct effect on mature neurons and glial cells (primary cultures). J Tissue Eng Regen Med 5, 668, 2011.

24. Meyerrose, T., Olson, S., Pontow, S., Kalomoiris, S., Jung, Y., Annett, G., et al. Mesenchymal stem cells for the sustained in vivo delivery of bioactive factors. Adv Drug Deliv Rev 62, 1167, 2010.

25. Martín-López, E., Nieto-Díaz, M., and Nieto-Sampedro, M. Differential adhesiveness and neurite-promoting activity for neural cells of chitosan, gelatin, and poly-L-lysine films. J Biomater Appl 26, 791, 2012.

26. Amaral, I.F., Cordeiro, A.L., Sampaio, P., and Barbosa, M.A. Attachment, spreading and short-term proliferation of human osteoblastic cells cultured on chitosan films with different degrees of acetylation. J Biomater Sci Polym Ed 18, 469, 2007.

27. Hamilton, V., Yuan, Y., Rigney, D.A., Puckett, A.D., Ong, J.L., Yang, Y., et al. Characterization of chitosan films and effects on fibroblast cell attachment and proliferation. Journal of materials science. Mater Med 17, 1373, 2006.

28. Yuan, Y., Zhang, P., Yang, Y., Wang, X., and Gu, X. The interaction of Schwann cells with chitosan membranes and fibers in vitro. Biomaterials 25, 4273, 2004.

29. López-Pérez, P.M., da Silva, R.M.P., Serra, C., Pashkuleva, I., and Reis, R.L. Surface phosphorylation of chitosan significantly improves osteoblast cell viability, attachment and proliferation. J Mater Chem 20, 483, 2010.

30. Ribeiro-Samy, S., Silva, N.A., Correlo, V.M., Fraga, J.S., Pinto, L., Teixeira-Castro, A., et al. Development and characterization of a PHB-HV-based 3D scaffold for a 
tissue engineering and cell-therapy combinatorial approach for spinal cord injury regeneration. Macromol Biosci 13, 1576, 2013.

31. Shea, G.K.H., Tsui, A.Y.P., Chan, Y.S., and Shum, D.K.Y. Bone marrow-derived Schwann cells achieve fate commitment - a prerequisite for remyelination therapy. Exp Neurol 224, 448, 2010.

32. Silva, N.A., Gimble, J.M., Sousa, N., Reis, R.L., and Salgado, A.J. Combining adult stem cells and olfactory ensheathing cells: the secretome effect. Stem Cells Dev 22, 1232, 2013.

33. Ladak, A., Olson, J., Tredget, E.E., and Gordon, T. Differentiation of mesenchymal stem cells to support peripheral nerve regeneration in a rat model. Exp Neurol 228, $242,2011$.
Address correspondence to: Antonio J. Salgado, PhD Life and Health Sciences Research Institute (ICVS) School of Health Sciences University of Minho Campus Gualtar 4710-057 Braga Portugal

E-mail: asalgado@ecsaude.uminho.pt

Received: October 2, 2013

Accepted: February 10, 2011

Online Publication Date: April 22, 2014 